\title{
Diagnosis of aortic stenosis in older age groups using external carotid pulse recording and phonocardiography
}

\author{
KATHLEEN H FLOHR,* E KENNETH WEIR, ELLIOT CHESLER \\ From the Medical Service, Cardiovascular Section, Veterans Administration Medical Center and the \\ University of Minnesota, Minneapolis, Minnesota, USA
}

SUMMARY Measurements made from carotid pulse tracings and phonocardiograms are helpful in the prediction of severe aortic stenosis in young patients. In the elderly, however, analysis of the carotid pulse tracing and, in particular, its maximum rate of rise has proved unreliable because the range of normal values for this age group has not been established. Consequently, we studied 44 young normal and 44 older normal subjects, 15 older patients with systolic hypertension, and 36 older patients with significant aortic stenosis. Measurements obtained from the carotid pulse included the left ventricular ejection time, upstroke time, half-rise time, and the maximum rate of rise. The peak of the systolic murmur in aortic stenosis was measured phonocardiographically from the $Q$ wave of the simultaneously recorded electrocardiogram (QP interval). The results confirm that the maximum rate of rise of the carotid pulse of normal older subjects is much more rapid than that of young subjects. Among elderly patients with aortic stenosis, the maximum rate of rise falls within the range of the normal young. Measurement of the half-rise time is imprecise, and there is considerable overlap with the normal. This is also true of left ventricular ejection time. The upstroke time is unreliable because of wave distortion produced by the arteriosclerotic vascular bed. The two most valuable and easily recorded measurements are the maximum rate of rise and the QP interval. A maximum rate of rise of more than $1000 \mathrm{mmHg} / \mathrm{s}$ and a QP interval of less than $220 \mathrm{~ms}$ argue strongly against the diagnosis of severe aortic stenosis. These measurements provide a simple, non-invasive means of avoiding left heart catheterisation in older subjects suspected of having severe aortic stenosis.

The clinical diagnosis of aortic stenosis in older patients is difficult. ${ }^{1-3}$ Systolic murmurs are common and may emanate from the aortic or mitral valves, or both. Aortic ejection murmurs may signify severe aortic stenosis or, equally well, haemodynamically insignificant thickening and calcification of the valve cusps. Frequently, these murmurs are heard better at the apex than at the base and may be confused with mitral regurgitation. The slow rising carotid pulse, a classic sign of aortic stenosis in the young, is often absent in the elderly because of diminished vascular compliance. Additionally, the symptoms of severe aortic stenosis such as heart failure, syncope, and angina pectoris are confusing in the older age group because they so commonly result from coronary artery or cerebrovascular disease. It is not surprising, therefore,

* Minnesota Heart Association Fellow.

Received for publication 11 December 1980 that left heart catheterisation is so frequently required to resolve this diagnostic dilemma. A sensitive and specific non-invasive test would be more desirable in the assessment of these patients.

In young patients, it is possible to determine the presence and severity of aortic stenosis from the external carotid pulse tracing and the phonocardiogram. ${ }^{4-6}$ In older patients, however, measurements made from the carotid pulse and, in particular, the maximum rate of rise, have proved to be unreliable because they have been compared with normal values obtained from the young and, in fact, the range of normal values for older subjects has not been established. In this study, therefore, external carotid pulse tracings and phonocardiograms obtained from patients over 50 years of age with aortic stenosis were compared with those from subjects in the same age group without apparent heart disease. 


\section{Methods}

External carotid pulse tracings were obtained using an air-coupled strain gauge transducer as described by Lewis et al., ${ }^{7}$ and tracings were recorded on photographic paper at a speed of $100 \mathrm{~mm} / \mathrm{s}$. Phonocardiograms were obtained using an Electronics for Medicine microphone with a centre frequency of 200 to 300 cycles per second.

External carotid pulse tracings and phonocardiograms were obtained in the following groups of patients:

Group 1 (young, normal medical students, hospital employees, and students): 27 male and 17 female, ages 14 to 47 (mean age 29) without evidence of heart disease.

Group 2 (old normal subjects): 44 men, ages 52 to 83 (mean age 65) without evidence of heart disease from history, physical examination, electrocardiogram, or chest $x$-ray film.

Group 3 (patients with systolic hypertension): 15 men, ages 61 to 87 (mean age 69 ) with systolic blood pressures of $150 \mathrm{mmHg}$ or greater. Some patients in this group had angina pectoris but none had evidence of cardiomegaly or congestive cardiac failure.

Group 4 (patients with moderate aortic stenosis): 16 men, ages 51 to 66 (mean age 59) without significant aortic regurgitation determined by angiography and with an aortic valve area determined by cardiac catheterisation of 0.7 to $1.0 \mathrm{~cm}^{2}$.

Group 5 (patients with severe aortic stenosis): 19 men and one woman, ages 50 to 82 (mean age 63 ) without significant aortic regurgitation determined by angiography and with an aortic valve area less than $0.7 \mathrm{~cm}$.

\section{ANALYSIS OF EXTERNAL CAROTID PULSE TRACINGS}

The measurements made from the carotid pulse tracings are shown diagrammatically in Fig. 1. The carotid pulse tracing was at least $5 \mathrm{~cm}$ in amplitude, and measurements were made from an average of 10 consecutive cycles. The left ventricular ejection time (LVET) was measured from the point of rise of the carotid pulse after the

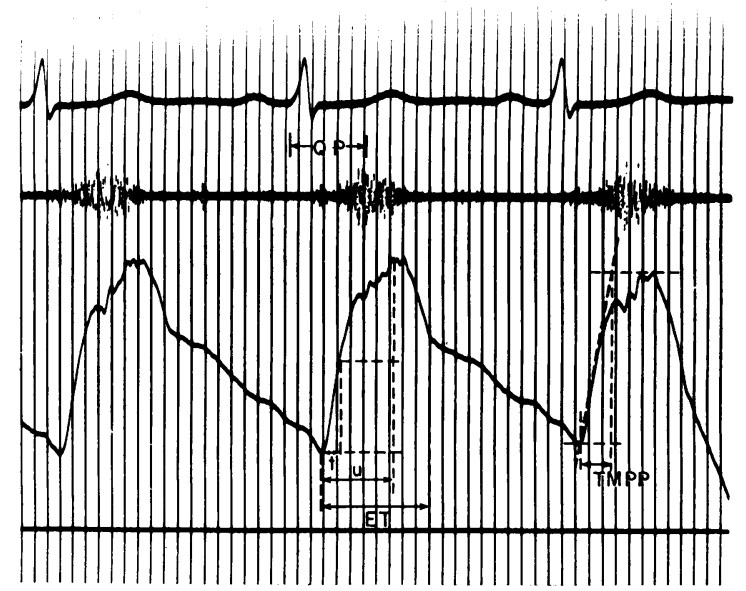

Fig. 1 Carotid artery and phonocardiographic tracings from a 63-year-old patient with aortic stenosis (valve area $0.5 \mathrm{~cm}^{2}$ ). Maximum rate of rise $=626 \mathrm{mmHg} / \mathrm{s}$, upstroke time $(u)=240$, half-rise time $(t)=42$, ejection time $(E T)=390,(Q P)=240$, all in ms. For details of measurements see text. TMPP $=$ time to maximum pulse pressure.

initial hump produced by isovolumic systole, to the dicrotic notch. The LVET was corrected for heart rate by the Bazett formula, ${ }^{8}$ dividing the measured LVET by the square root of the RR interval to obtain LVET $_{c}$, and by the Weissler formula by adding 1.7 times the heart rate in men, or 1.6 times the heart rate in women, to the measured LVET to obtain LVET $_{\mathrm{w} .}{ }^{9}$ The upstroke time was measured from the carotid upstroke to the peak of the carotid pulse tracing. The half-rise time was measured from the beginning of the carotid upstroke to the half maximum point on the carotid pulse curve. Both half-rise and upstroke times were corrected for heart rate using the Bazett formula. The maximum rate of rise in $\mathrm{mmHg} / \mathrm{s}$ was obtained by dividing the brachial artery pulse pressure (obtained by sphygmomanometry) by the time required to reach the peak of the carotid trace along a line tangent to the carotid upstroke at its steepest point.

Table Effect of age, hypertension, and aortic stenosis on carotid pulse tracings

\begin{tabular}{|c|c|c|c|c|c|c|c|}
\hline \multicolumn{2}{|c|}{ Group } & \multirow{2}{*}{$\begin{array}{l}\begin{array}{l}\text { Maximum rate } \\
\text { of rise }(\mathrm{mmHg} / \mathrm{s})\end{array} \\
920 \pm 32 \\
1272 \pm 53 \\
1763 \pm 160 \\
593 \pm 55 \\
565 \pm 47\end{array}$} & \multirow{2}{*}{$\begin{array}{l}\text { LVETC } \\
(m s)\end{array}$} & \multirow{2}{*}{$\begin{array}{l}\begin{array}{l}L V E T w \\
(m s)\end{array} \\
416 \pm 9 \\
405 \pm 4 \\
421 \pm 23 \\
429 \pm 4 \\
448 \pm 4\end{array}$} & \multirow{2}{*}{$\begin{array}{l}\begin{array}{l}\text { Upstroke time } \\
(m s)\end{array} \\
119 \pm 7 \\
120 \pm 10 \\
190 \pm 10 \\
202 \pm 11 \\
215 \pm 10\end{array}$} & \multirow{2}{*}{$\begin{array}{l}\begin{array}{l}\text { Half-rise time } \\
(\mathrm{ms})\end{array} \\
35 \pm 1 \\
32 \pm 2 \\
32 \pm 2 \\
56 \pm 7 \\
56 \pm 5\end{array}$} & \multirow{2}{*}{$\begin{array}{c}\begin{array}{c}Q P \text { interval } \\
(m s)\end{array} \\
\\
\\
234 \pm 11 \\
270 \pm 6\end{array}$} \\
\hline $\begin{array}{l}1 \\
2 \\
3 \\
4 \\
5\end{array}$ & $\begin{array}{l}\text { Young normal (44) } \\
\text { Old normal (44) } \\
\text { Old hypertensive (15) } \\
\text { Moderate AS (16) } \\
\text { Severe AS }(20)\end{array}$ & & & & & & \\
\hline
\end{tabular}

$\star$ Values listed are mean \pm standard error of the mean. 
ANALYSIS OF PHONOCARDIOGRAMS

Previous work has shown that the timing of the peak of the systolic murmur is a sensitive index of the severity of aortic stenosis, whereas the length of the murmur has little predictive value. ${ }^{10}$ Therefore, we determined the peak of the crescendo of the systolic murmur in at least five cycles and measured this distance from the preceding $Q$ wave of the simultaneously recorded electrocardiogram (QP interval).

\section{Results}

Measurements obtained from carotid pulse tracing and phonocardiograms in patient groups 1 to 5 are shown in the Table. Values for the maximum rate of rise in these patient groups are shown in Fig. 2. The mean value for the old normals (group 2) (1272 \pm 53$)($ SEM) was higher than the value obtained in the young normals (group 1) $(920 \pm 32)$. Group 5 (severe aortic stenosis) had a maximum rate of rise of $565 \pm 47$ and showed considerable overlap with the young normals (group 1), but had a significantly lower maximum rate of rise than old normals (group 2) $(p<0.001)$. No patient with severe aortic stenosis had a maximum rate of rise over $1000 \mathrm{mmHg} / \mathrm{s}$ (Fig. 2). A maximum rate of rise of less than $1000 \mathrm{mmHg} / \mathrm{s}$ had a 95 per cent sensitivity and 92 per cent

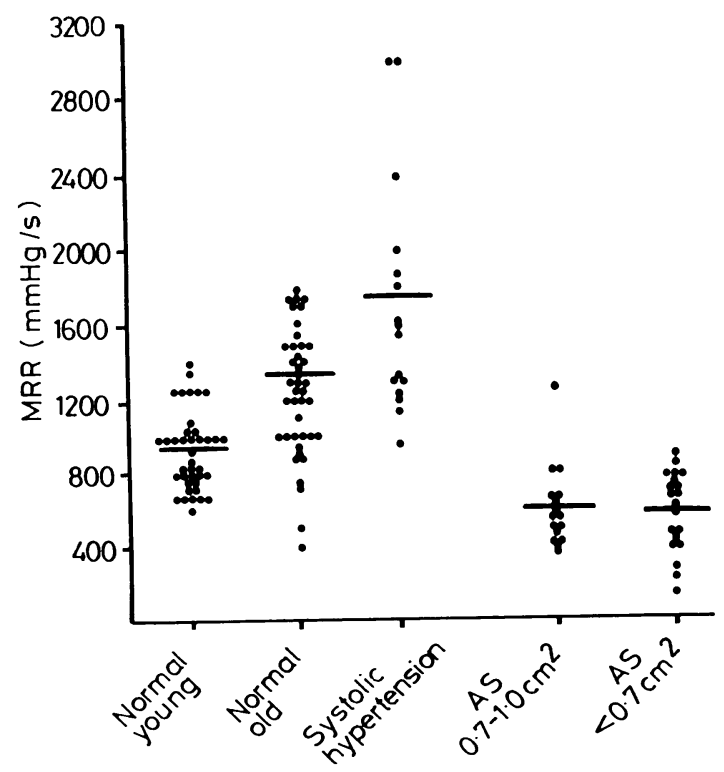

Fig. 2 Scattergram comparing the maximum rate of rise of the carotid pulse (MRR) in the five groups studied. The horizontal bar, in this and Fig. 3 to 6, indicates the mean and standard error of the mean.

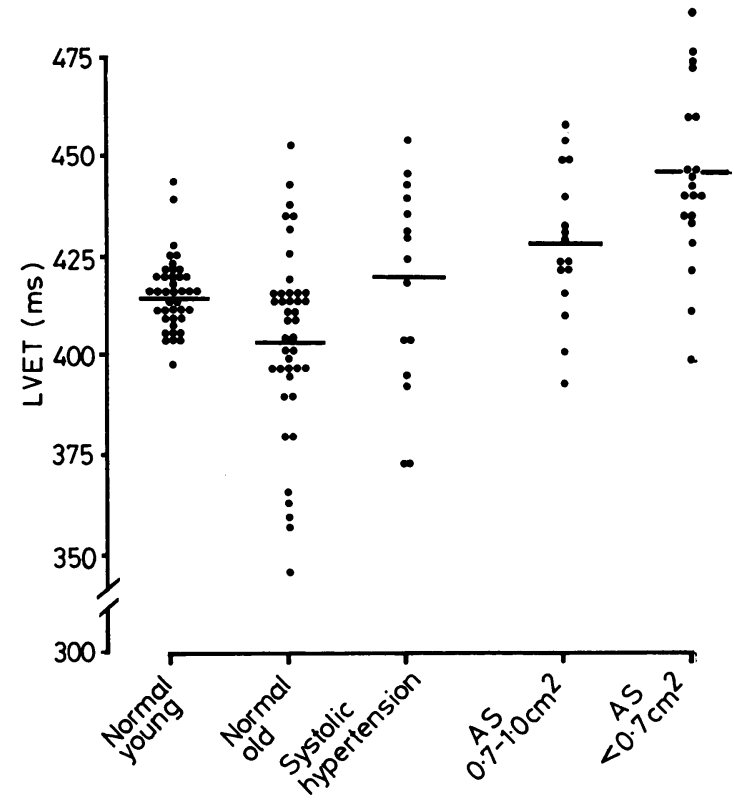

Fig. 3 Scattergram comparing the left ventricular ejection time $\left(L V E T_{w}\right)$ in the five groups studied.

specificity in detecting moderate or severe aortic stenosis. Among the older patients with aortic stenosis (groups 4 and 5), there was no significant correlation between aortic valve area and the maximum rate of rise $(r=0 \cdot 20)$.

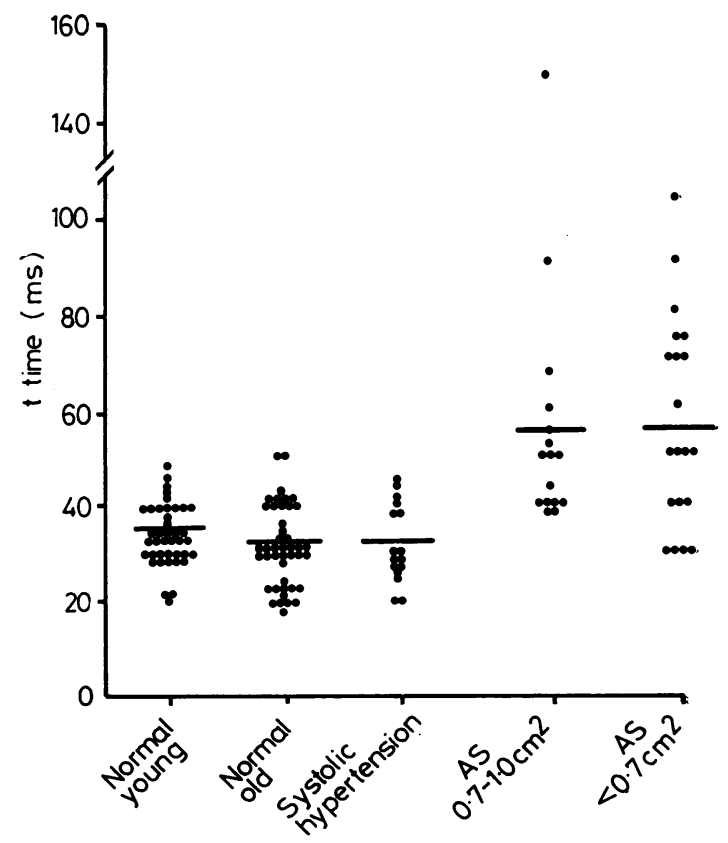

Fig. 4 Scattergram comparing the half-rise (t) times. 
The values for LVET in groups 1 to 5 are shown in Fig. 3. The mean value for the $\mathrm{LVET}_{w}$ in the young normals (group 1) of $416 \pm 9 \mathrm{~ms}$ was virtually the same as the figure of $413 \pm 10 \mathrm{~ms}$ obtained by Weissler et al. ${ }^{11}$ The mean LVET $_{w}$ for the old patients with severe aortic stenosis was significantly longer than for their age matched controls (group 2) $(\mathrm{p}<0.001)$. When the older patients with moderate and severe aortic stenosis were combined, LVET $_{w}$ correlated with aortic valve area $(p<0.01)$.

The half-rise times of old normal subjects and those with aortic stenosis (groups 2 and 5) were also significantly different $(p<0.01)$, but we found measurement of this narrow interval relatively imprecise at the paper speed $(100 \mathrm{~mm} / \mathrm{s})$ used for our tracings (Fig. 4).

The values for upstroke time in groups 1 to 5 are shown in Fig. 5. The time for group 2 was significantly lower than that for group $5(\mathrm{p}<0.001)$ but there was no significant difference between the patients with aortic stenosis and those with systolic hypertension. In the older patients, the upstroke time in the hypertensive group was much longer than in the normotensives. No patient with severe aortic stenosis had an upstroke time below $100 \mathrm{~ms}$. Within groups 4 and 5, there was no significant correlation between upstroke time and valve area $(\mathrm{r}=0 \cdot 38)$.

The interval from the onset of the QRS complex of the electrocardiogram to the peak of the systolic murmur recorded on the phonocardiogram (QP) in all patients with aortic stenosis (groups 4 and 5) is shown in Fig. 6. No patient with severe aortic

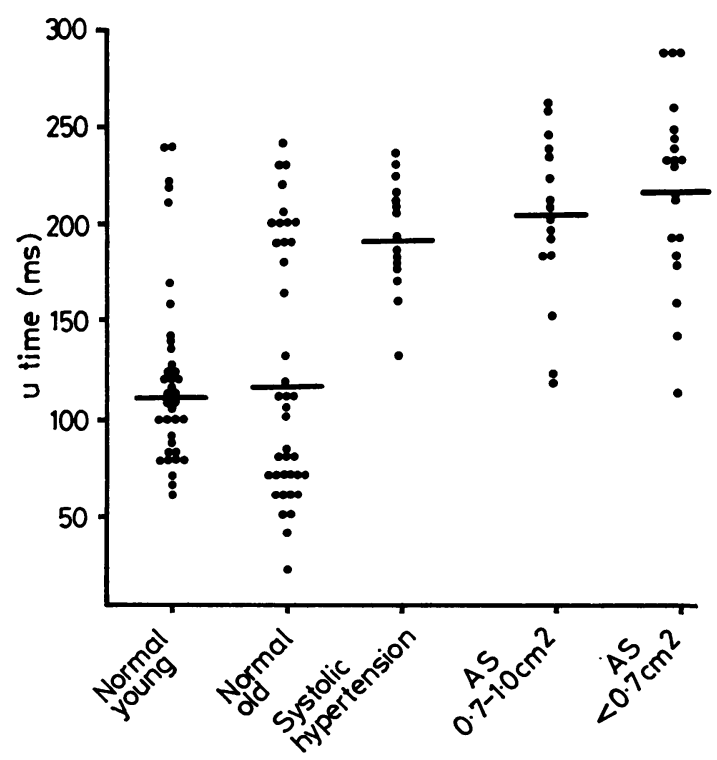

Fig. 5 Scattergram comparing the upstroke (u) times.

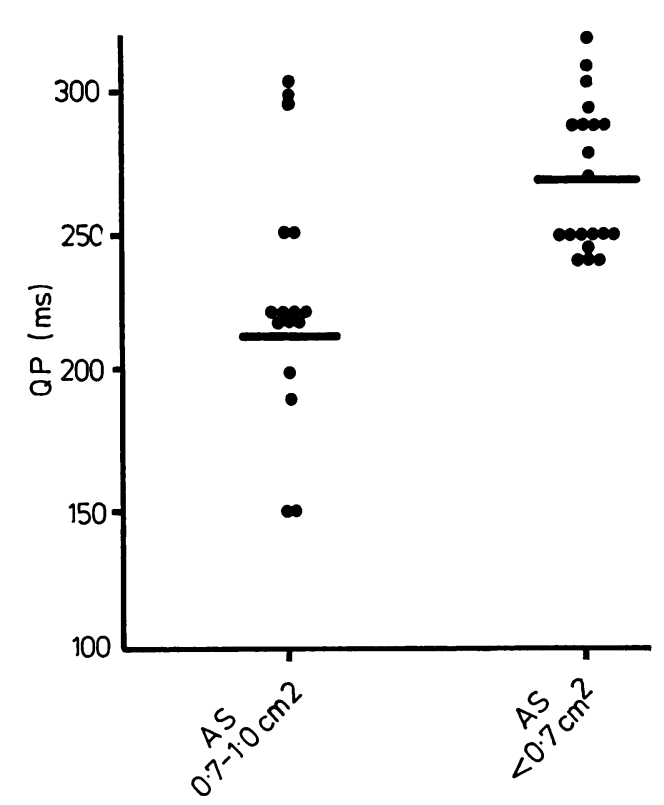

Fig. 6 Scattergram comparing the QP intervals obtained from the phonocardiogram in patients with aortic stenosis.

stenosis had a QP value less than $220 \mathrm{~ms}$. QP values correlated with aortic valve area $(r=0.59, \mathrm{p}<0.01)$, as shown in Fig. 6.

\section{Discussion}

Slowing of the carotid upstroke in young patients is a reliable clinical sign of haemodynamically significant aortic stenosis. In the elderly patient with severe aortic stenosis, however, the carotid upstroke is deceptively brisk, presumably because of diminished vascular compliance. ${ }^{3}$ Bonner et al. ${ }^{6}$ studied a group of patients with significant aortic stenosis in the age range 23 to 69 years and found that a maximum rate of rise of less than $400 \mathrm{mmHg} / \mathrm{s}$ was highly suggestive of severe aortic stenosis. In five of their patients, however, aged 58 to 69 years, the maximum rate of rise was over $500 \mathrm{mmHg} / \mathrm{s}$. Lyle et al. ${ }^{12}$ studied a group of patients of unstated age with aortic stenosis (not necessarily severe) and found that 88 per cent had a maximum rate of rise less than $639 \mathrm{mmHg} / \mathrm{s}$.

Our study shows that older normal subjects have a higher maximum rate of rise than young normal subjects. Furthermore, older patients with aortic stenosis may have a maximum rate of rise which falls within the normal range for the young. Normal values determined for the maximum rate of rise in young subjects, therefore, are inapplicable to the elderly. It is apparent from our data, however, 
that a maximum rate of rise in excess of 1000 $\mathrm{mmHg} / \mathrm{s}$ makes the diagnosis of moderate and severe aortic stenosis in the elderly patient very unlikely.

Acceleration of the upstroke of the carotid artery is not the only distortion that occurs with advancing age. The initial systolic maximum of the carotid pulse (percussion wave) occurs simultaneously with the peak velocity of aortic blood flow and corresponds with the anacrotic bend of the pulse. After the anacrotic bend, during the period of diminished velocity of aortic blood flow, the second systolic maximum (tidal wave) is inscribed on the carotid pulse tracing and is usually associated with peak aortic pressure. This late systolic rise is a result of pulsatile flow in distensible tubes with impended run-off. When the latter is accentuated because of increased peripheral resistance, as occurs in hypertensive patients, the tidal wave is increased. Thus, an increase in the amplitude of the tidal wave has been induced by vasoconstrictor and diminished by vasodilator drugs. ${ }^{13}$ The effect of a prominent delayed tidal wave in systolic hypertension is to prolong considerably the upstroke time into the
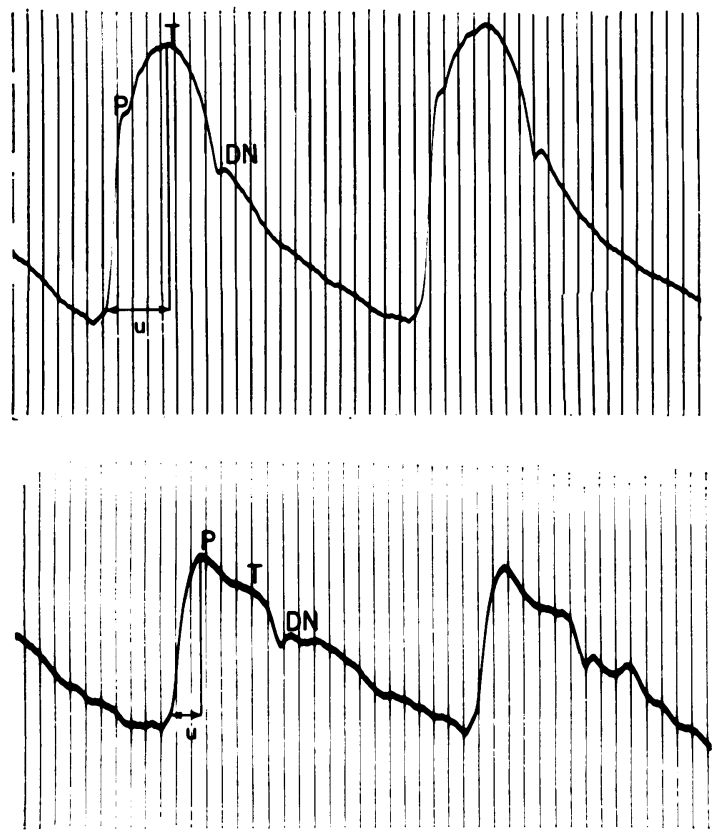

Fig. 7 Carotid tracings obtained from an elderly subject with systolic hypertension (BP 200/98 $\mathrm{mm} \mathrm{Hg}$ ) (top) and from a young normal control (bottom). The rate corrected upstroke $(u)$ time for the young adult is $107 \mathrm{~ms}$ whereas that for the elderly hypertensive is $180 \mathrm{~ms}$ (see text). $P=$ percussion wave; $T=$ tidal wave; $D N=$ dicrotic notch. range encountered in aortic stenosis (Fig. 5 and 7). Additionally, there appeared to be a bimodal distribution for the upstroke time in the older normotensives with one group centred around $80 \mathrm{~ms}$ and the other around $200 \mathrm{~ms}$, a value similar to that found in systolic hypertensives. The prolonged upstroke time in the older normotensives is probably a result of diminished vascular compliance in the elderly atherosclerotic patient. The upstroke time is thus of little diagnostic value in the diagnosis of aortic stenosis.

Lengthening of the LVET in aortic stenosis has been reported, but it may shorten with the onset of cardiac failure. ${ }^{14} \mathrm{Nesje}^{15}$ found no significant correlation between LVET and aortic valve gradient in his patients with aortic stenosis. In our patients, the LVET was significantly shorter in the old normal group than in the group with aortic stenosis, but the overlap between the groups was considerable.

The half-rise time has been found to correlate significantly with the aortic valve gradient. ${ }^{15}$ In our patients, the half-rise time was significantly longer in aortic stenosis compared with controls, but we found this interval difficult to measure precisely even at a paper speed of $100 \mathrm{~mm} / \mathrm{s}$. In addition, like the ejection time, the overlap between the various groups was considerable.

Phonocardiographically, it has been clearly shown by Oakley and Hallidie-Smith ${ }^{10}$ that the severity of the aortic valve gradient correlates well with the timing of the peak of the murmur-the later the peak, the more severe the stenosis. Bonner et al. ${ }^{6}$ confirmed these findings and showed that innocent systolic murmurs peak at a QP measurement of $187 \pm 15 \mathrm{~ms}$ and this may overlap with mild aortic stenosis; a QP of $200 \mathrm{~ms}$, however, indicated severe aortic stenosis. None of our patients with severe aortic stenosis had a QP of less than $220 \mathrm{~ms}$.

Using the measurements obtained in this study, a maximum rate of rise in excess of $1000 \mathrm{mmHg} / \mathrm{s}$ makes the diagnosis of severe aortic stenosis unlikely. Combining this with a phonocardiographic measurement of a QP interval of less than $220 \mathrm{~ms}$ provides a non-invasive screening test for excluding severe aortic stenosis in the older patient.

\section{References}

1 Finegan RE, Gianelly RE, Harrison DC. Aortic stenosis in the elderly; relevance of age to diagnosis and treatment. $N$ Engl $\mathcal{F}$ Med 1969; 281: 1261-4.

2 Perez GL, Jacob M, Bhat PK, Rao DB, Luisada AA. Incidence of murmurs in the aging heart. $\mathcal{F} \mathrm{Am}$ Geriatr Soc 1976; 24: 29-31. 
3 Crowley IS, Morris DC, Silverman BD. Valvular heart disease. In: Hurst JW, Logue RB, Schlant RC, Wenger NK, eds. The heart, arteries, and veins. 4th ed. New York: McGraw-Hill, 1978: 1038-9.

4 Robinson B. The carotid pulse. I. Diagnosis of aortic stenosis by external recordings. Br Heart $\mathcal{f}$ 1963; 25 : 51-60.

5 Epstein EJ, Coulshed N. Assessment of aortic stenosis from the external carotid pulse wave. $\mathrm{Br}$ Heart $\mathcal{f}$ 1964; 26: 84-96.

6 Bonner AJ, Jr, Sacks HN, Tavel ME. Assessing the severity of aortic stenosis by phonocardiography and external carotid pulse recordings. Circulation 1973; 48: $247-52$.

7 Lewis RP, Rittgers SE, Forester WF, Boudoulas H. A critical review of the systolic time intervals. Circulation 1977; 56: 146-58.

8 Bazett HC. An analysis of the time-relations of electrocardiograms. Heart 1920; 7: 353-70.

9 Weissler AM, Harris LC, White GD. Left ventricular ejection time index in man. $f$ Appl Physiol 1963; 18: 919-23.

10 Oakley CM, Hallidie-Smith KA. Assessment of site and severity in congenital aortic stenosis. $\mathrm{Br}$ Heart $\mathcal{f}$ 1967; 29: 367-79.

11 Weissler AM, Harris WS, Schoenfeld CD. Systolic time intervals in heart failure in man. Circulation 1968; 37: 149-59.

12 Lyle DP, Bancroft WH, Jr, Tucker M, Eddleman $\mathrm{EE}, \mathrm{Jr}$. Slopes of the carotid pulse wave in normal subjects, aortic valvular diseases, and hypertrophic subaortic stenosis. Circulation 1971; 43: 374-81.

13 Freis ED, Heath WC, Luchsinger PC, Snell RE. Changes in the carotid pulse which occur with age and hypertension. Am Heart f 1966; 71: 757-65.

14 Tavel ME. Clinical phonocardiography and external pulse recording. 2nd ed. Chicago: Year Book Medical Publishers, 1972.

15 Nesje OA. Severity of aortic stenosis assessed by carotid pulse recordings and phonocardiography. Acta Med Scand 1978; 204: 321-30.

Requests for reprints to Dr Elliot Chesler, Veterans Administration Medical Center, 54th Street and 48th Avenue South, Minneapolis, Minnesota 55417, USA. 